\title{
TRIBUTE TO THE REVIEWERS OF THE \\ CANADIAN JOURNAL OF HOSPITAL PHARMACY
}

The Canadian Society of Hospital Pharmacists and the Editorial Board of the Canadian Journal of Hospital Pharmacy (CJHP) would like to thank the following people for serving as peer reviewers for the CJHP in 2020. Their assistance has helped us continue to ensure the high quality of articles published in the Journal.

Shirin Abadi
Margaret Louise Ackman
Elissa Shing Yuk Aeng
Yazid Nabih Al Hamarneh
Loyd Allen
Alison Alleyne
Mayce Al-Sukhni
Barb Angel
Michael Armas
Hoan Linh Banh
Arden Barry
Bill Bartle
Marisa Battistella
Marie France Beauchesne
Alysha Bharmal
Lisa D Bishop
Emily K Black
Philippe Bouchard
Denis Brouillette
Paula Brown
Cenzina Caligiuri
Roxane Rae Carr
April Chan
Feng Chang
Flay Charbonneau
Lori Chen
Ivy Chow
Jocelyne Chretien
Celine Corman
Sue Corrigan
Dennis Cote
Sylvain Côté
Renée Dagenais
Karen Dahri
Dawn Dalen
Bruce Dalton
Carolyn Dittmar

\begin{tabular}{l} 
Awissi Don-Kelena \\
Anar Dossa \\
Douglas Doucette \\
Linda Dresser \\
Marc Alexandre Duceppe \\
Zack Jules Dumont \\
Wasim S El Nekidy \\
Patrick Fitch \\
Jean-Marc Forest \\
Marie-André Fournier \\
Rochelle M Gellatly \\
Alfred Gin \\
Sean K Gorman \\
Carolyn Gray \\
Micheal Guirguis \\
Nathan Hann \\
Curtis K Harder \\
Ayesha Hassan \\
Jennifer Hawkes \\
Philippe Hogan \\
Roxanne Hook \\
Sherilyn K D Houle \\
Tony Howarth \\
Lauren M J Hutton \\
Marianne F Ivey \\
Lawrence Jackson \\
Rochelle Marie Johnston \\
Andrea Kent \\
Mark George Klang \\
Alicia Koo \\
Sheri L Koshman \\
Ines Krass \\
Daniel James Lalor \\
Elaine Lau \\
Gilles Leclerc \\
Anna Lee \\
Colin Lee \\
\hline
\end{tabular}

Michael Legal
Derek Leong
Elizabeth Leung
Marianna Leung
Timothy Leung
Valerie Leung
Vivian W Y Leung
Anita Lo
Peter S Loewen
Stephanie Lovering
Alan Low
Janice Ma
Vincent H Mabasa
John R Manderville
Judith Marin
Dan Martinusen
Gilbert Matte
Karen McDermaid
Rumi McGloin
Erin McIsaac
Susan McKenna
Bruce Millin
Mits Miyata
Janice Munroe
Tania Mysak
Jeff Nagge
Lynne Nakashima
Heather L Neville
Cesilia Nishi
Claire O’Reilly
Asad Patanwala
Jonathan Penm
Christine Stephanie Peragine
Jerrold L Perrott
Leah Pritchett
Taylor Raiche
Eric J P Romeril
Sila

Marc Romney

Myrella Roy

Cheryl A Sadowski

Humera Sarwar

Theresa J Schindel

Amir Anthony Shahidi

Catherine Sicard

Dan Sitar

Jacky T P Siu

Sean P Spina

Beth Sproule

Melanie Anne Sunderland

Najla Tabbara

Zoé Thiboutot

Peter Thomson

Kathryn Timberlake

James E Tisdale

Angela Tong

Christine Truong

Ricky D Turgeon

Nisha Varughese

Andrew Veysey

Philippe D Vincent

Scott E Walker

Karen Walsh

Richard G Wanbon

Caroline Warnock

Jennifer Wiebe

Kyle John Wilby

David R Williamson

William Wong

Deborah Yoong

Jamie Zao

Maria Zhang

Jian Song Zhou

Rosemary Zvonar

(née Piquette)

The continuing support that these peer reviewers demonstrate for our publication is greatly appreciated, and we thank them for their time and effort on behalf of the CJHP.

We would like to take this opportunity to ask reviewers to renew their commitment to the CJHP's peer review process by re-registering through our new online submission and peer review system at: https://cjhp-online.ca/index.php/cjhp/user/register. Please ensure the Reviewer box is checked on your profile. You may also update your reviewing interests, so our editors can correctly match you with appropriate submissions. On this registration page, you can also register as an author by checking the Author box on your profile, should you wish to submit manuscripts for consideration by the CJHP in the future. 